\title{
Analysis of the Effects of Applying PDCA to Keeping Detailed Medical Records during Standardized Resident Training
}

\author{
Hongwei Wang*, Wei Fu, Wenyan Yuan and Kaijian Xia \\ Changshu Hospital Affiliated to Soochow University, First People's Hospital of Changshu City, \\ Changshu 215500, Jiangsu Province, China \\ 1040558124@qq.com \\ *The corresponding author
}

Keywords: PDCA; Sstandardized residenttraining; Pass rate

\begin{abstract}
Objective: To discuss PDCA application in the management of keeping detailed medical records during standardized resident training. Method: By obtaining the truth rate of medical record registration through their retrospective data collection during standardized training of residents enrolled in 2015. Result: By way of PDCA analysis, reasons are found for the problems that exist in detailed medical records kept by residents, relevant rectification measures are implemented, and finally the quality of detailed medical records kept by residents is improved. Conclusion: PDCA application in detailed medical records kept by residents can improve the truth and pass rate, thus improving the quality of standardized resident training.
\end{abstract}

\section{Introduction}

Standardized resident training is an important part of post-graduate education of medical students. It is extremely important for the training of high-level clinicians and the improvement of medical care quality. It occupies an important position in medical life-long education that comes before continuing medical education and after basic education in medical schools. It is the key to forming medical clinical experts. PDCA cycle is an important way to improve quality management and is the basic way to ensure the high-speed operation of quality systems. It also applies to standardized resident training. ${ }^{[1][2]}$

Since 1996, standardized staff resident training has been conducted in our hospital in accordance with the "Standardized Training Programs of Clinical Residents" by the Science and Technology Education Division of the Ministry of Health, "Programs for Continuing Education of Residents in County Hospitals "by the Health and Family-planning Commission of Jiangsu Province, and the relevant provisions by the Health and Family-planning Commission of Changshu City. Our hospital has undertaken the standardized training tasks. At present, qualified residents total 435. PDCA cycle has been adopted to intervene in the training quality of residents enrolled in 2015. Suggestions areput forward on establishing and improving scientific quality control systems for standardized resident training in the region through preliminary system research, effect evaluation and cause analysis. 


\section{Study the Reasons for Standardized Resident Training Using PDCA Cycle}

1.1 Standardized resident training is a complex systematic program and an industrial chain in "producing" clinicians. This "product" has in one such functions as the learning of comprehensive medical theories, medical policies and regulations and diagnosis and treatment technology, the treatment of complex medical problems. Diversified teaching methods and combined multi-sectoral actions need to be adopted to complete it. With regulations and norms of various levels in it and influenced by a large number of subjective and objective factors, it is a complex and diversified system. Therefore, only through applying to scientific management concepts to monitor each link can the quality standardization of standardized resident training as a whole be ensured in the implementation of the entire industrial chain.

1.2 PDCA cycle, which is a mature quality management theory applicable to the medical field, is first proposed by the American quality management expert William Edward Deming, and is also known as "Deming cycle". It includes 4 stages of plan-do-check-action. Being a procedural, standardized and scientific management method and applicable to all quality management processes, it can improve work quality in continuous cycles. In the PDCA theory are put forward scientific process methods and basic elements which the establishment of quality control system should follow. This theory is widely used in various fields of quality management practice and achieved good results. Compared with other modern enterprise management theories and methods, PDCA cycle has been widely used in the field of science, and has played an important role in the fields of clinical path, continuous medical quality improvement, clinical teaching management, nursing teaching management, pharmacy teaching management and so on. Therefore, this method has been well known and highly acceptable to hospital managers ${ }^{[3-8]}$. In 2016, "Research on the Application of PDCA Cycle in Improving Standardized Training Quality in Hospitals" was approved by the Changshu municipal government as the annual science and technology program of 2016 funded by the Health and Family-planning Commission of Changshu City.

\section{Plan}

The main purpose of this process is to investigate, analyze and diagnose the current status of the training of health service workers of the No. 1 Changshu People's Hospital, to grasp the main factors that affect staff training quality, to determine the guidelines and objectives of standardized resident training, and to formulate work plans for all the hospital staff. As early as the 1990s, the Health and Family-planning Commission of Changshu Cityset up the post-graduate medical education committee, which studied, guided, and managed the post-graduate medical education work of the whole city, co-ordinate the planning, standards and norms of the standardized resident training. To guarantee the provision of working staff for establishing a basic medical and health system covering urban and rural areas, it promulgated "the Implementation Plan for Changshu Standardized Resident Training Work (trial)", which clarified the organization structure, work content, enrollment plan, training mode and guaranteeing measures. It also formulated "Administrative Measures for identifying Changshu Standardized Resident Training Bases (trial)", "Standards for Changshu Standardized Resident Training Bases (trial)", "General Rules for Changshu Standardized Resident Training Standards (trial)." 
Between January 2016 and December 2016, PDCA cycle was usedinour hospital to improve the truth and pass rate of medical records by trainees who received standardized residenttraining. Relevant results are reported as follows:

\section{Do}

Object of Research. Object of research: the truth rate ofregistered detailed medicalrecords by trainees who were enrolled in 2015 andreceived standardized residenttraining. Numeratordefinition: the number of registered detailed medical records which were kept by residents enrolled in 2015 and which met the requirements in monitoring periods.Denominator definition: the total of detailed medical records by residents enrolled in 2015 in monitoring periods. Monitoring time interval: from January 2016 to December 2016.

Method of Research. According to national standards of standardized residenttraining, it is necessary to ensure the authenticity of training, detailed medical records inputted by residents to the Jiangsu provincial standardizedresident training management platform should contain personal signatures. All case numbers of detailed medical records inputted by trainees enrolled in 2015 were retrieved from the platform during monitoring and were systematically sampled. And these records were checked after being retrieved from medical record rooms. Monitoring was conducted monthly in our hospital.Systematic sampling method was used to carry out retrospective data collection. The total sample size was 50-300.

\section{Check}

Truth Rate Check. The truth rate of registered detailed medical records by trainees who were enrolled in 2015 and received standardized resident training between January 2016 and December 2016werechecked by trained professionals, with the time from January to April 2016as a baseline survey. Data verification formula: $100 \%$ - | verifieddata - original data |/ original data * $100 \%$. Results are as follows:

\begin{tabular}{|c|c|c|c|c|c|c|c|c|c|}
\hline Month & 16.04 & 16.05 & 16.06 & 16.07 & 16.08 & 16.09 & 16.10 & 16.11 & 16.12 \\
\hline $\begin{array}{c}\text { Numer } \\
\text { anom }\end{array}$ & 24 & 48 & 11 & 42 & 55 & 38 & 50 & 32 & 26 \\
\hline $\begin{array}{c}\text { Denom } \\
\text { Result }\end{array}$ & $\begin{array}{c}64 \\
m\end{array}$ & 102 & 35 & 43 & 62 & 38 & 52 & 32 & 26 \\
\hline $\begin{array}{c}4 \\
m\end{array}$ & $\begin{array}{c}47.06 \\
m\end{array}$ & $\begin{array}{c}97.67 \\
m\end{array}$ & $\begin{array}{c}88.71 \\
m\end{array}$ & $100 \%$ & $\begin{array}{c}96.15 \\
m\end{array}$ & $100 \%$ & $100 \%$ \\
\hline
\end{tabular}

Note: Numeratordefinition: the number of registered detailed medical records which were kept by residents enrolled in 2015 and which met the requirements in monitoring periods.Denominator definition: the total of detailed medical records by residents enrolled in 2015 in monitoring periods. 


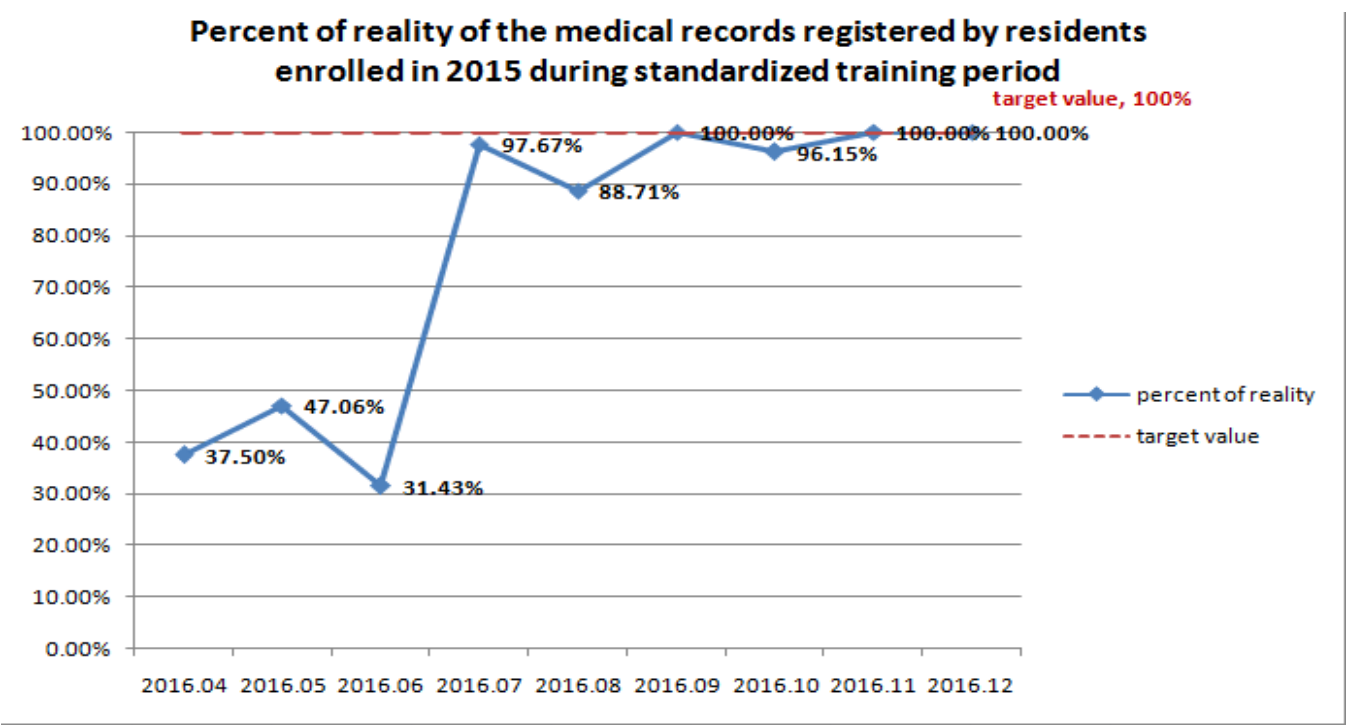

Figure 1. Finite The truth rate of registered detailed medical records by residents enrolled in 2015 who received standardized training

Analysis of Causes. Analyses of causes of low truth rate in April 2016: (1) Trainees did not fill in as required. (2) In-patient medical records were kept in the electronic medical record (EMR) system, and were not printed out in archived medical records. (3) Admission records and in-patient medical records cannot co-exist in the new EMR system, resulting in the inability of in-patient medical records to be kept. (4) Part of the residents were not aware that their accounts can be logged on in the EMR to fill in medical records. Teachers' accounts were used to log in when keeping medical records. (5) Rotary departments did not require in-patient medical records to be kept. Analysis of causes of low truth rate between May 2016 to June 2016: After the low truth rate in April 2016 was recognized, improving measures began to be implemented on May 3rd, 2016. Medical records registered between 2016.1.1 to 2016.2.29 were checked in May 2016.Medical records registered between January 2016 and April 2016 were inputted to the system before the implementation of improving measures, hence the inability of these record sto show the effectiveness of PDCA measures.

\section{Action}

Rectification Measures. (1)Resident seminars were held, reasons were found out, and detailed medical record keeping requirements were reiterated. (2) Co-ordination with the information section and modification of the new version of the EMR system, enabled the co-existence of in-patient medical and admission records. (3) Resident accounts and usage of the EMR system were publicized at "Teaching and Research" column on the intranet. (4) Communications with teachers were conducted, and the need was stressed for trainees to keep detailed medical records.

\section{Conclusion}

After carrying out improving measures using PDCA, medical record truth rate checking resultsin 
July 2016 is shown in Figure 1.Registered medical record truth rate rose significantly and was close to the target value. The truth rate in August fell compared with the previous month, with a total of 7 failed medical records. It was learned through communication that not enough attention was paid by relevant trainees. Through communication with relevant trainees individually, it was reiterated that it was important for "detailed medical records to carry personal signatures". The pass rate in October fell to some degree. Through communication with relevant trainees, it was learned that detailed medical records were actually kept by trainees and that only a few departments, such as the department of urology, printed admissions instead of putting the detailed medical records kept by trainees into the case history. Contacts with relevant department directors and teachers told them that it was necessary to put detailed medical records kept by trainees into the case history. Since November 2016, the truth rate has all been 100\%, improving measures have been obviously effective, and monitoring is still going on.

\section{Discussion}

PDCA cycle is a basic method of running the total quality management system. Its effective implementation is based on scientific analysis of large amount of data. Comprehensive use can be made of various management techniques and methods, with particular emphasis on step-by-step advancement and continuous innovative development of management work. The application of PDCA cycle in this paper to the management of the whole process of standardized training has optimized the training management process, clarified the responsibilities of staff from various departments, seized the key links and steps, and achieved the continuous improvement of resident training in dynamic operations and better implementation effects.

\section{Acknowledgements}

Fund Project: The financing of the project of Changshu City health and Family Planning Commission (Fund number: csws201620)

\section{References}

[1] Peijie L I, Liu X. THE APPLICATION OF PDCA IN STANDARDIZED TRAINING OF RESIDENT DOCTORS[J]. Medical Journal of Qilu, 2014.

[2] Xue J, Fan L, Wu W. Application and discussion of PDCA cycle in the training management of resident doctors in Department of orthopedics[J]. Medical Journal of the Present Clinical, 2016.

[3] Liu J, Jianliang D U, Liao N. THE APPLICATION OF PDCA CIRCULATION METHOD ON THE ARCHIVING MANAGEMENT OF MEDICAL RECORDS[J]. Modern Hospital, 2014.

[4] Fu G, Zhou A, Huang L. The application of PDCA cycle in a large number of pre-service standardized training of new nurses[J]. Medical Forum, 2013.

[5] Wang B, He K, Wang J. Application of PDCA combined with PIO health education model to the follow-up of patients with standardized treatment of cancer pain in the service demonstration ward[J]. Journal of Qilu Nursing, 2014. 
[6] Chen Y K. Study and Application of PDCA Circulation to Quality Standardized Building in Coal Enterprise[J].

[7] Tye M J, Wheeler W E. Applying FOCUS-PDCA Methodology to Imrpove Patient Meal Service Practices[J]. Journal of the American Dietetic Association, 2007, 107(8):A71-A71.

[8] Guo F D, Equipment D O. Discussion on the Training of Medical Devices Clinical Application Based on PDCA Method[J]. China Medical Devices, 2014.

[9] Liu Y J, Tian Z C, Han M, et al. Discussion on Application of PDCA Theory in Metal and Non-Metal Mine Safety Standardization Management System[J]. Applied Mechanics \& Materials, 2013, 357-360:2590-2593.

[10] Xu M, Liang X, Tan M, et al. Application of PDCA cycle in training HELPERR procedures for handling shoulder dystocia[J]. Journal of Nursing Science, 2011.

[11] Makoto Matsuo, Jun Nakahara. The effects of the PDCA cycle and OJT on workplace learning[J]. The International Journal of Human Resource Management, 2013, 21(1):195-207.

[12]Feehery P A, Allen S, Bey J. Flushing 101: Using a FOCUS-PDCA Quality Improvement Model to Reduce Catheter Occlusions with Standardized Protocols[J]. Journal of Vascular Access Devices, 2003, 8(2):38-45. 